\title{
Homoclinic Bifurcation of a Quadratic Family of Real Functions with Two Parameters
}

\author{
Salma M. Farris, Karam N. Abdul-Kareem \\ Department of Mathematics, College of Computer and Mathematical Sciences, University of Mosul, Mosul, Iraq \\ Email: Salmaalfaris925@gmail.com, Karamck13s4@gmail.com
}

How to cite this paper: Farris, S.M. and Abdul-Kareem, K.N. (2021) Homoclinic Bifurcation of a Quadratic Family of Real Functions with Two Parameters. Open Access Library Journal, 8: e7300.

https://doi.org/10.4236/oalib.1107300

Received: March 10, 2021

Accepted: May 28, 2021

Published: May 31, 2021

Copyright $\odot 2021$ by author(s) and Open Access Library Inc.

This work is licensed under the Creative Commons Attribution International License (CC BY 4.0).

http://creativecommons.org/licenses/by/4.0/

\section{Abstract}

In this work the homoclinic bifurcation of the family $H=\left\{h_{a, b}(x)=a x^{2}+b: a \in \mathbb{R} /\{0\}, b \in \mathbb{R}\right\}$ is studied. We proved that this family has a homoclinic tangency associated to $x=0$ of $P_{1}$ for $b=\frac{-2}{a}$. Also we proved that $W^{u}\left(P_{1}\right)$ does not intersect the backward orbit of $P_{1}$ for $b>\frac{-2}{a}$, but has intersection for $b<\frac{-2}{a}$ with $a>0$. So $H$ has this type of the bifurcation.

\section{Subject Areas}

Dynamical System

\section{Keywords}

Local Unstable Set, Unstable Set, Homoclinic Point, Homoclinic Orbit, Non-Degenerate, Homoclinic Tangency, Homoclinic Bifurcation

\section{Introduction}

There are various definitions for the homoclinic bifurcation. In the sense of Devaney, the homoclinic bifurcation is a global type of bifurcations, that is, this type of bifurcation is a collection of local and simple types of bifurcations [1] (like, period-doubling and saddle-node of bifurcation [2]).

According to [3] [4] [5] we have another definition of the homoclinic bifurcation via the notions of the unstable sets of a repelling periodic point (fixed point) and the intersection of this set with the backward orbits of this point.

The purpose of this work is to prove the family

$H=\left\{h_{a, b}(x)=a x^{2}+b: a \in \mathbb{R} /\{0\}, b \in \mathbb{R}\right\}$ has homoclinic bifurcation at $b=\frac{-2}{a}$ 
following the second definition.

\section{Definitions and Basic Concepts}

\subsection{Definition 1: [6]}

A fixed point $P$ is said to be expanding for a map $f$, if there exists a neighborhood $U(P)$ such that $\left|f^{\prime}(x)\right|>1$ for any $x \in U(P)$.

The neighborhood in the previous definition is exactly the local unstable set.

\subsection{Definition 2: [7]}

Let $P$ be a repelling fixed point for a function $f: I \rightarrow I$ on a compact interval $I \subset R$, then there is an open interval about $P$ on which $f$ is one-to-one and satisfies the expansion property. $|f(x)-f(P)|>|x-P|, \forall x \in I$ where $x \neq P$.

The interval in the previous definition is exactly the unstable set of $P$.

\subsection{Definition 3: [8]}

Let $P$ is fixed point and $f^{\prime}(P)>1$ for a map $f: \mathbb{R} \rightarrow \mathbb{R}$. A point $q$ is called homoclinic point to $P$ if $q \in w_{l o c}^{u}(P)$ and there exists $n>0$ such that $f^{n}(q)=P$.

\subsection{Definition 4: [9]}

The union of the forward orbit of $q$ with a suitable sequence of preimage of $q$ is called the homoclinic orbit of $P$. That is

$O(q)=\left\{P, \cdots, q_{-2}, q_{-1}, q, q_{1}, q_{2}, \cdots, q_{m}=P\right\} \quad$ where $q_{i+1}=f\left(q_{i}\right)$ for $i \leq m-1$, $q_{m}=P$ and $\lim _{i \rightarrow-\infty} q_{i}=P$.

\subsection{Definition 5: [10]}

The critical $x$ point is non-degenerate if $f^{\prime \prime}(x) \neq 0$. The critical point $x$ is degenerate if $f^{\prime \prime}(x)=0$.

\subsection{Definition 6: [11]}

Let $f$ be a smooth map on $I \subset R$, and let $p$ be a hyperbolic fixed point for the map $f$. If $W^{u}(p)$ intersects the backward orbit of $p$ at a nondegenerate critical point $x_{c r}$ of $f$, then $x_{c r}$ is called a point of homoclinic tangency associated to $p$.

\subsection{Definition 7: [3]}

Let $f_{\varphi}$ be a smooth map on $I \subset R$, and let $p$ be a hyperbolic fixed point for a map $f_{\varphi}$. We say that $f_{\varphi}$ has homoclinic bifurcation associated to $p$ at $\varphi=\hat{\varphi}$ if:

1) For $\varphi<\hat{\varphi}(\varphi>\hat{\varphi}), W^{u}(p)$ and the backward orbit of $p$ has no intersect.

2) For $\varphi=\hat{\varphi}, f_{\hat{\varphi}}$ has a point of homoclinic tangency $x_{c r}$ associated to $p$.

3) For $\varphi>\hat{\varphi}(\varphi<\hat{\varphi})$, the intersection of $W^{u}(p)$ with the backward orbit of $p$ is nonempty. 


\section{Homoclinic Bifurcation of the Family}

$$
H=\left\{\boldsymbol{h}_{a, b}(x)=a x^{2}+b: a \in \mathbb{R} /\{0\}, b \in \mathbb{R}\right\}
$$

In this section, we show that the family $H$ has a point of homoclinic tangency associated to $P_{1}$ at $b=\frac{-2}{a}$, and $H$ has a homoclinic bifurcation.

We need the following results proved in [12].

At the first, the fixed points of $h_{a, b}(x)$ are

$$
P_{1}=\frac{1+\sqrt{1-4 a b}}{2 a}, P_{2}=\frac{1-\sqrt{1-4 a b}}{2 a} .
$$

\section{a) Proposition:}

For $h_{a, b}(x) \in H$ with $a>0$ the local unstable set of the fixed point $P_{1}$ is $w_{l o c}^{u}\left(P_{1}\right)=\left(\frac{1}{2|a|}, \infty\right)$.

\section{b) Lemma:}

For $h_{a, b}(x) \in H, h_{a, b}^{-1}\left(P_{1}\right)=\mp \sqrt{\frac{P_{1}-b}{a}}=\mp P_{1}$ where $P_{1}>b$ for $a>0$.

c) Theorem:

For $h_{a, b}(x) \in H$ with $a>0$, the unstable set of the fixed point $P_{1}$ is $w^{u}\left(P_{1}\right)=\left(\frac{1}{|a|}-P_{1}, \infty\right)$.

d) Remark: [13]

The local unstable set of the fixed point $P_{2}$ is $w_{l o c}^{u}\left(P_{2}\right)=\left(-\infty, \frac{-1}{2|a|}\right)$, and the unstable set of the fixed point $P_{2}$ is $w^{u}\left(P_{2}\right)=\left(-\infty, \frac{-1}{|a|}-P_{2}\right)$. In this work we will omit the result about $P_{2}$ because $\left(h_{a, b}^{\prime}\left(P_{2}\right)<-1\right.$, when $b<\frac{-3}{4 a}$ for $a>0$ $b>\frac{-3}{4 a}$ for $a<0$ ). Thus we will not care for the fixed point $P_{2}$. (See definition (2.3)).

e) Remark:

For $h_{a, b}(x) \in H, h_{a, b}^{-2}\left(P_{1}\right)=\mp \sqrt{\frac{-P_{1}-b}{a}}$.

\section{f) Proposition:}

For $h_{a, b} \in H$, if $b<\frac{-(5+2 \sqrt{5})}{4 a}$ with $a>0$, then the second preimage of the fixed point $P_{1}$ belongs to the local unstable set of $P_{1}$.

g) Proposition:

For $h_{a, b} \in H$, if $\frac{-(5+2 \sqrt{5})}{4 a} \leq b \leq \frac{-2}{a}$ with $a>0$, then the third preimage 
of the fixed point $P_{1}$ belongs to the local unstable set of $P_{1}$.

\section{h) Theorem:}

For the family $H=\left\{h_{a, b}(x)=a x^{2}+b: a>0\right\}$, there exist homoclinic points to the fixed point $P_{1}$ whenever $b \leq \frac{-2}{a}$. Moreover $h_{a, b}^{-2}\left(P_{1}\right)=q_{1,1}, h_{a, b}^{-3}\left(P_{1}\right)=q_{2,1}$ are the first homoclinic points for $b<\frac{-(5+2 \sqrt{5})}{4 a}, \frac{-(5+2 \sqrt{5})}{4 a} \leq b \leq \frac{-2}{a}$ respictivelity.

\section{i) Example:}

For $h_{1,-6}(x)=x^{2}-6$, a homoclinic orbit of a homoclinic point $\sqrt{3}$ is: $O(\sqrt{3})=\{3,-3, \sqrt{3}, \cdots, 3\}$.

\section{The main result:}

\subsection{Lemma 1}

For $h_{a, b}(x)=a x^{2}+b$ with $a \in \mathbb{R} /\{0\}$, the critical point of $h_{a, b}(x)$ is 0 , and it is a non-degenerate critical point.

\section{Proof:}

Clearly that the critical point of $h_{a, b}(x)$ is zero.

Since $a \neq 0$, then

$$
h_{a, b}^{\prime \prime}(x)=2 a \neq 0 .
$$

So $h_{a, b}(x)$ has a non-degenerate critical point at $x=0$.

\subsection{Lemma 2}

If $b=0$ of $h_{a, b}(x) \in H$ with $a \in \mathbb{R} /\{0\}$, then the backward orbit of the repelling fixed point $P_{1}$ is undefined in $\mathbb{R}$.

Proof:

$h_{a, 0}(x)=a x^{2}$, clearly $P_{1}=\frac{1}{a}$.

Now the first preimage of $h_{a, 0}(x)$ is

$h_{a, 0}^{-1}(x)=\mp \sqrt{\frac{x}{a}}$, where $x>0$ for $a>0$.

By Lemma (3-b), we have

$$
h_{a, 0}^{-1}\left(\frac{1}{a}\right)=\mp \sqrt{\frac{1}{a^{2}}}=\mp \frac{1}{a}=\mp P_{1} .
$$

But $+P_{1}$ is a fixed point, and $-P_{1}=-\frac{1}{a} \notin w_{l o c}^{u}\left(P_{1}\right)=\left(\frac{1}{2 a}, \infty\right)$, see Proposition (3-a).

By Remark (3-e) we have

$$
h_{a, 0}^{-2}\left(P_{1}\right)=\mp \sqrt{\frac{-P_{1}}{a}}=\mp \sqrt{\frac{-\frac{1}{a}}{a}}=\mp \sqrt{\frac{-1}{a^{2}}} \notin \mathbb{R},
$$


since $\frac{1}{a^{2}}>0, \forall a \in \mathbb{R} /\{0\}$.

Therefore $h_{a, 0}^{-n}\left(P_{1}\right)$ are undefined in $\mathbb{R}$ with $n \geq 2$.

Thus the backward orbit of the repelling fixed point $P_{1}$ is undefined in $\mathbb{R}$

\subsection{Theorem 1}

For the family $h_{a, b}(x)=a x^{2}+b, 0$ belong to the backward orbit of $P_{1}$ whenever $b=\frac{-2}{a}$ with $a \in \mathbb{R} /\{0\}$, and the backward orbit of $P_{1}$ is:

$$
h_{a, \frac{-2}{a}}^{-n}\left(P_{1}=\frac{2}{a}\right)=\left\{\frac{2}{a},-\frac{2}{a}, 0, \frac{\sqrt{2}}{a}, \cdots\right\} .
$$

\section{Proof:}

We test the values of $n$ which makes $h_{a, b}^{-n}\left(P_{1}\right)=0$.

By Lemma (3-b), $h_{a, b}^{-1}\left(P_{1}\right)= \pm P_{1}$.

So $h_{a, b}^{-1}\left(P_{1}\right) \neq 0$.

Now suppose that $h_{a, b}^{-2}\left(P_{1}\right)=0$, by Remark (3-e) then $h_{a, b}^{-2}\left(P_{1}\right)=\mp \sqrt{\frac{-P_{1}-b}{a}}=0$, thus

$$
\begin{gathered}
\frac{-P_{1}-b}{a}=0 \\
-P_{1}-b=0 \\
P_{1}=b .
\end{gathered}
$$

Since the fixed point $P_{1}=\frac{1+\sqrt{1-4 a b}}{2 a}$, therefore

$$
\frac{1+\sqrt{1-4 a b}}{2 a}=-b,
$$

then

$$
\begin{gathered}
1+\sqrt{1-4 a b}=-2 a b \\
\sqrt{1-4 a b}=-2 a b-1 \\
1-4 a b=4 a^{2} b^{2}+4 a b+1
\end{gathered}
$$

$4 a^{2} b^{2}+8 a b=0$, which implies

$4 a b(a b+2)=0$, then either

$b=0$, but by the above Lemma (3.2) the backward orbit of $P_{1}$ is undefined, so we omit this case.

Or $a b+2=0$, thus

$$
b=\frac{-2}{a} .
$$

Now, $P_{1}=\frac{2}{a}$ and to find the backward orbit of $P_{1}$, we consider

$$
h_{a, \frac{2}{a}}^{-1}(x)= \pm \frac{\sqrt{a x+2}}{a} .
$$


By Lemma (3-b) $h_{a, b}^{-1}\left(P_{1}\right)= \pm P_{1}$, then $h_{a, \frac{-2}{a}}^{-1}\left(\frac{2}{a}\right)= \pm \frac{2}{a}$. But $+\frac{2}{a}$ is a fixed point, therefore

$$
h_{a, \frac{-2}{a}}^{-1}\left(\frac{2}{a}\right)=-\frac{2}{a} \text {. }
$$

So

$$
\begin{gathered}
h_{a, \frac{-2}{a}}^{-2}\left(\frac{2}{a}\right)=\frac{\sqrt{a\left(\frac{-2}{a}\right)+2}}{a}=0 . \\
h_{a, \frac{-2}{a}}^{-3}\left(\frac{2}{a}\right)=\frac{\sqrt{a(0)+2}}{a}=\frac{\sqrt{2}}{a}, \text { and so on. }
\end{gathered}
$$

Therefore the backward orbit of $P_{1}=\frac{2}{a}$ is:

$$
h_{a, \frac{-2}{a}}^{-n}\left(P_{1}=\frac{2}{a}\right)=\left\{\frac{2}{a},-\frac{2}{a}, 0, \frac{\sqrt{2}}{a}, \cdots\right\} .
$$

\subsection{Example}

For $h_{1,-2}(x)=x^{2}-2,0$ belongs to the backward orbit of $P_{1}=2$ (Figure 1), and the backward orbit of $P_{1}$ is $h_{1,-2}^{-n}(2)=\{2,-2,0, \sqrt{2}, \cdots, 2\}$.

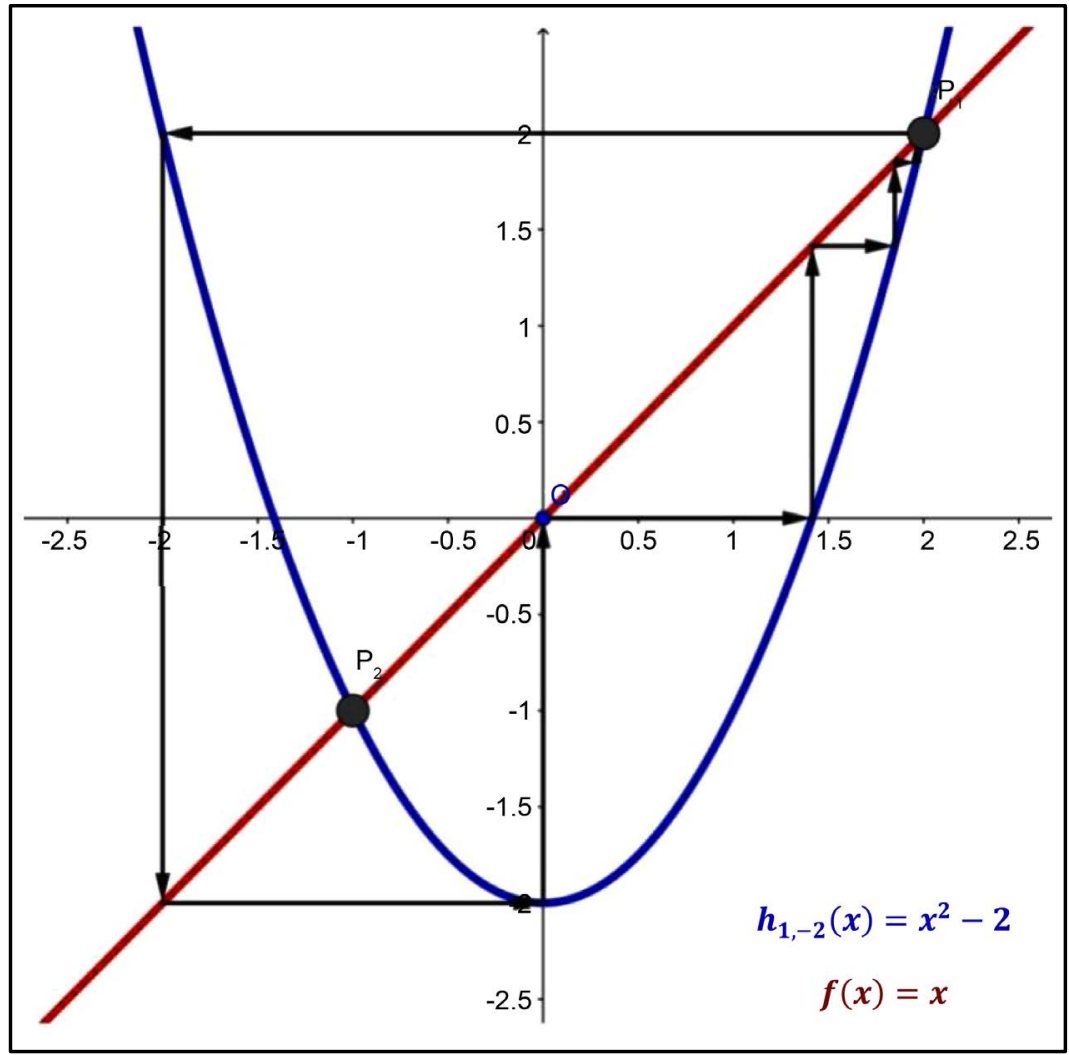

Figure 1. For $h_{1,-2}(x)=x^{2}-2$, the backward orbit of $P_{1}=2$. 


\subsection{Theorem 2}

If $b>\frac{-2}{a}$ for $h_{a, b}(x) \in H$ with $a>0$, then there is no intersection of the backward orbit with the unstable set of $P_{1}$.

Proof:

The backward orbit of $P_{1}$

By Lemma (3-b) $h_{a, b}^{-1}\left(P_{1}\right)= \pm P_{1}$, since $+P_{1}$ is a fixed point, then we consider

$$
h_{a, b}^{-1}\left(P_{1}\right)=-P_{1} \text {. }
$$

By Remark (3-e), $h_{a, b}^{-2}\left(P_{1}\right)=\mp \sqrt{\frac{-P_{1}-b}{a}}$.

If $-P_{1}>b$, then by Theorem (3-h),

$b \leq \frac{-2}{a}$ which is a contradiction with $b>\frac{-2}{a}$. Therefore

$-P_{1}<b$, which implies

$$
h_{a, b}^{-2}\left(P_{1}\right) \notin \mathbb{R} .
$$

So $h_{a, b}^{-n}\left(P_{1}\right)$ are undefined in $\mathbb{R}$ with $n \geq 2$.

Thus the backward orbit of $P_{1}$ is undefined .

So the intersection of $W^{u}\left(P_{1}\right)$ with the backward orbit of $P_{1}$ is also undefined.

\subsection{Theorem 3}

If $b=\frac{-2}{a}$ for $h_{a, b}(x) \in H$ with $a>0$, then $h_{a, \frac{-2}{a}}$ has a point of homoclinic tangency at 0 associated to $P_{1}$.

Proof:

By Theorem (3.3), $h_{a, \frac{-2}{a}}^{-n}\left(P_{1}=\frac{2}{a}\right)=\left\{\frac{2}{a},-\frac{2}{a}, 0, \frac{\sqrt{2}}{a}, \cdots\right\}$.

By Theorem $(3-c), W^{u}\left(P_{1}\right)=\left(\frac{1}{a}-P_{1}, \infty\right)$, then

$$
\begin{aligned}
& W^{u}\left(P_{1}=\frac{2}{a}\right)=\left(\frac{1}{a}-\frac{2}{a}, \infty\right), \text { i.e. } \\
& W^{u}\left(P_{1}=\frac{2}{a}\right)=\left(-\frac{1}{a}, \infty\right) . \text { Now } \\
& h_{a, \frac{-2}{a}}^{-n}\left(\frac{2}{a}\right) \text { intersects } W^{u}\left(P_{1}=\frac{2}{a}\right) \text { at } 0 .
\end{aligned}
$$
By Lemma (3.1) 0 is a non-degenerate critical point. So $h_{a, \frac{-2}{a}}$ has a point of
homoclinic tangency at 0 associated to $P_{1}$.

\subsection{Theorem 4}

If $b<\frac{-2}{a}$ for $h_{a, b}(x) \in H$ with $a>0$, then the backward orbit of $P_{1}$ crosses the unstable set $W^{u}\left(P_{1}\right)$. 


\section{Proof:}

First consider the backward orbit of $P_{1}$.

By Lemma (3-b) $h_{a, b}^{-1}\left(P_{1}\right)= \pm P_{1}$.

But $+P_{1}$ is a fixed point, therefore we consider

$$
h_{a, b}^{-1}\left(P_{1}\right)=-P_{1} \text {. }
$$

By Remark (3-e), $h_{a, b}^{-2}\left(P_{1}\right)=\mp \sqrt{\frac{-P_{1}-b}{a}}$.

Since $b<\frac{-2}{a}$, then by Theorem (3-h)

$$
h_{a, b}^{-2}\left(P_{1}\right) \in \mathbb{R} .
$$

Let $h_{a, b}^{-2}\left(P_{1}\right)=q_{1,1}, h_{a, b}^{-3}\left(P_{1}\right)=q_{2,1}$.

By Proposition (3-f), if $b<\frac{-(5+2 \sqrt{5})}{4 a}$, then $q_{1,1} \in W_{\text {loc }}^{u}\left(P_{1}\right)$.

By Proposition (3-g), if $\frac{-(5+2 \sqrt{5})}{4 a} \leq b<\frac{-2}{a}$, then $q_{2,1} \in W_{\text {loc }}^{u}\left(P_{1}\right)$.

Now since the local unstable set of the repelling fixed point contained in the unstable set of the repelling fixed point. Therefore

$$
h_{a, b}^{-n}\left(P_{1}\right) \cap W^{u}\left(P_{1}\right) \neq \varnothing .
$$

Following examples explain the cases for $b>\frac{-2}{a}, b=\frac{-2}{a}$ and $b<\frac{-2}{a}$ (with $a>0$ ) respectively.

\subsection{Example 1}

For $h_{1,-1}(x)=x^{2}-1$, we have no intersection of the backward orbit of $P_{1}$ with the unstable set of $P_{1}$.

\section{Solution:}

Consider the fixed point of $h_{1,-1}(x)$ is $P_{1}=\frac{1+\sqrt{5}}{2}$, and

$$
h_{1,-1}^{-1}(x)= \pm \sqrt{x+1} .
$$

The backward orbit of $P_{1}=\frac{1+\sqrt{5}}{2}$

$$
\begin{aligned}
& h_{1,-1}^{-1}\left(\frac{1+\sqrt{5}}{2}\right)= \pm \frac{1+\sqrt{5}}{2} \text {, where }+\frac{1+\sqrt{5}}{2} \text { is a fixed point, therefore we consider } \\
& h_{1,-1}^{-1}\left(\frac{1+\sqrt{5}}{2}\right)=-\frac{1+\sqrt{5}}{2} \text {. Now } \\
& h_{1,-1}^{-2}\left(\frac{1+\sqrt{5}}{2}\right)=\mp \sqrt{-\frac{1+\sqrt{5}}{2}+1} \notin \mathbb{R} .
\end{aligned}
$$

So $h_{1,-1}^{-n}\left(\frac{1+\sqrt{5}}{2}\right)$ are undefined in $\mathbb{R}$ with $n \geq 2$. 
Thus the backward orbit of $P_{1}$ is undefined.

So the intersection of $W^{u}\left(\frac{1+\sqrt{5}}{2}\right)$ with the backward orbit of $P_{1}$ is also undefined.

\subsection{Example 2}

For $h_{1,-2}(x)=x^{2}-2$, then $h_{1,-2}$ has a point of tangency at 0 associated to $P_{1}$.

\section{Solution:}

Consider the fixed point of $h_{1,-2}(x)$ is $P_{1}=2$.

By Example (3.4), The backward orbit of $P_{1}=2$ is

$$
h_{1,-2}^{-n}(2)=\{2,-2,0, \sqrt{2}, \cdots, 2\} .
$$

On the other hand, the unstable set of $P_{1}=2$ is

$W^{u}(2)=(-1, \infty)$, (see Theorem (3-c)). Now

$h_{1,-2}^{-n}(2)$ intersects $W^{u}(2)$ at 0 .

By Lemma (3.1), 0 is a non-degenerate critical point. So $h_{1,-2}$ has a point of tangency at 0 associated to $P_{1}=2$.

\subsection{Example 3}

For $h_{1,-6}(x)=x^{2}-6$, the backward orbit of $P_{1}$ crosses the unstable set $W^{u}\left(P_{1}\right)$.

Solution:

First consider the fixed point $P_{1}=3$.

The backward orbit of 3 is:

$h_{1,-6}^{-n}(3)=\{3,-3, \sqrt{3}, \cdots, 3\} \quad$ (see Example (3-i)), with

$h_{1,-6}^{-1}(3)=h_{1,-2}^{2}(\sqrt{3})$, and $h_{1,-6}^{-2}(3)=\sqrt{3}$.

Since $\sqrt{3}$ is a homoclinic point of $P_{1}=3$, then

$$
\sqrt{3} \in W_{l o c}^{u}(3) \text {. }
$$

Now since the local unstable set of the repelling fixed point $P_{1}=3$ contained in the unstable set of the repelling fixed point $P_{1}=3$. Therefore

$$
h_{1,-6}^{-n}(3) \cap W^{u}(3) \neq \varnothing \text {. }
$$

Note, the main theorem in the work:

\subsection{Theorem 5}

$h_{a, b}(x)=a x^{2}+b, a>0$ has a homoclinic bifurcation associated to the repelling fixed point of $h_{a, b}, P_{1}=\frac{1+\sqrt{1-4 a b}}{2 a}$, at $b=\frac{-2}{a}$.

\section{Proof:}

1) For $b>\frac{-2}{a}$, by Theorem (3.5) the intersection of the backward orbit of $P_{1}$ and the unstable set of $P_{1}$ is undefined.

2) For $b=\frac{-2}{a}$, by Theorem (3.6) $h_{a, \frac{-2}{a}}$ has a point of homoclinic tangency 
associated to $P_{1}$ at $x=0$.

3) For $b<\frac{-2}{a}$, by Theorem (3.7) the backward orbit of $P_{1}$ crosses the unstable set of $P_{1}, W^{u}\left(P_{1}\right)$.

Therefore $h_{a, b}$ has a homoclinic bifurcation associated to $P_{1}$ at $b=\frac{-2}{a}$.

\subsection{Example}

$h_{1,-2}(x)=x^{2}-2$ has a homoclinic bifurcation associated to the repelling fixed point of $h_{1,-2}, P_{1}=2$, at $b=-2$.

$h_{1,-2}(x)$ has a homoclinic bifurcation associated to the repelling fixed point of $h_{1,-2}, P_{1}=2$, at $b=-2$. See examples (3.8), (3.9), (3.10).

\subsection{Remark}

For $a<0$, we have same results which proved above for $a>0$. In fact, we can prove in similar ways, that: $h_{a, b}(x)=a x^{2}+b, a<0$ has a homoclinic bifurcation associated to the repelling fixed point of $h_{a, b}, P_{1}=\frac{1+\sqrt{1-4 a b}}{2 a}$, at $b=\frac{-2}{a}$.

\section{Conclusion}

We conclude that the family $H=\left\{h_{a, b}(x)=a x^{2}+b: a \in \mathbb{R} /\{0\}, b \in \mathbb{R}\right\}$ has homoclinic tangency associated to $P_{1}$ at the critical point $x=0$. Also for $b>\frac{-2}{a}$ we have no intersection between the backward orbit of $P_{1}$ and the unstable set of $P_{1}$, and the backward orbit of $P_{1}$ crosses the unstable set of $P_{1}$ for $b<\frac{-2}{a}$. So we have homoclinic bifurcation at $b=\frac{-2}{a}$.

\section{Conflicts of Interest}

The authors declare no conflicts of interest regarding the publication of this paper.

\section{References}

[1] Zhusubaliyev, Z.T. and Mosekilde, E. (2003) Bifurcations and Chaos in Piecewise-Smooth Dynamical Systems. World Scientific, Singapore. https://doi.org/10.1142/5313

[2] Ghrist, R.W., Holmes, P.J. and Sullivan, M.C. (2006) Knots and Links in Three-Dimensional Flows. Springer, Berlin Heidelberg.

[3] Onozaki, T. (2018) Nonlinearity, Bounded Rationality, and Heterogeneity. Springer, Berlin Heidelberg. https://doi.org/10.1007/978-4-431-54971-0

[4] Guo, S. and Wu, J. (2013) Bifurcation Theory of Functional Differential Equations, Vol. 10. Springer, New York. https://doi.org/10.1007/978-1-4614-6992-6

[5] Puu, T. and Sushko, I. (2006) Business Cycle Dynamics. Springer, New York. https://doi.org/10.1007/3-540-32168-3 
[6] Block, L.S. and Coppel, W.A. (2006) Dynamics in One Dimension. Springer, New York.

[7] Devaney, R. (2018) An Introduction to Chaotic Dynamical Systems. CRC Press, Boca Raton, FL. https://doi.org/10.4324/9780429502309

[8] Gulick, D. (2012) Encounters with Chaos and Fractals. CRC Press, Boca Raton, FL. https://doi.org/10.1201/b11855

[9] Laura, G., Viktor, A., Iryna, S. and Fabio, T. (2019) Continuous and Discontinuous Piecewise-Smooth One-Dimensional Maps: Invariant Sets and Bifurcation Structures, Vol. 95. World Scientific, Singapore.

[10] Perko, L. (2013) Differential Equations and Dynamical Systems, Vol. 7. Springer Science \& Business Media, Berlin Heidelberg.

[11] Bonatti, C., Díaz, L.J. and Viana, M. (2006) Dynamics beyond Uniform Hyperbolicity: A Global Geometric and Probabilistic Perspective, Vol. 102. Springer Science \& Business Media, Berlin Heidelberg.

[12] Abdul-Kareem, K.N. and Farris, S.M. (2020) Homoclinic Points and Homoclinic Orbits for the Quadratic Family of Real Functions with Two Parameters. Open Access Library Journal, 7, 1-18. https://doi.org/10.4236/oalib.1106170

[13] Abdul-Kareem, K.N. and Farris, S.M. (2020) Homoclinic Points and Bifurcation for a Quadratic Family with Two Parameters. MSc Thesis, Department of Mathematics, College of Computer and Mathematical Sciences, University of Mosul, Mosul, Iraq, 1-98. 